\title{
5, 10-methylenetetrahydrofolate reductase gene mutation and reproductive outcome: how much do we know? A case series in Indian population
}

\author{
Pooja Nadkarni Singh, Mridula Raghav*, Prabhakar Singh, Vanita Viradiya
}

Nimaaya Women's Center for Health, Surat, Gujarat, India

Received: 27 December 2021

Revised: 07 February 2022

Accepted: 08 February 2022

\section{*Correspondence:}

Dr. Mridula Raghav,

E-mail: drmridularaghav@ hotmail.com

Copyright: (c) the author(s), publisher and licensee Medip Academy. This is an open-access article distributed under the terms of the Creative Commons Attribution Non-Commercial License, which permits unrestricted non-commercial use, distribution, and reproduction in any medium, provided the original work is properly cited.

\section{ABSTRACT}

Recurrent pregnancy loss (RPL) defined as loss of two or more pregnancies is one of the reasons why couples visit an ART clinic. 2-5\% of RPL cases show an abnormal parental karyotype. Folic acid is an essential B vitamin involved in processes of fundamental importance for cell division and embryo development. Folic acid deficiency can have effect on many processes involved in oocyte development, acquisition of endometrial receptivity, embryo implantation and also in the maintenance of pregnancy. The 5, 10-methylenetetrahydrofolate reductase (MTHFR) enzyme plays an important role in folate metabolism. The most investigated MTHFR gene mutations are single nucleotide polymorphisms (SNPs) at the mRNA positions 677 (rs1801133) and 1298 (rs1801131). MTHFR gene mutations are found less in Asian population and hence have not been studied or evaluated much. We presented a case series of 3 different cases of MTHFR gene mutation variants that were managed at our ART center. Testing for MTHFR gene mutation after 3 early pregnancy failures/missed abortions, PGT-A and surrogacy being our proposed interventions.

Keywords: MTHFR gene mutation, RPL, Recurrent IVF failures, Surrogacy, MTHFR c.677C>T, MTHFR c.1298A>C

\section{INTRODUCTION}

Folic acid is an important B vitamin essential for human reproduction. ${ }^{3}$ The processing of folic acid and other dietary folates is vital for many key processes such as amino acid metabolism, purine and pyrimidine synthesis and methylation of nucleic acids, proteins and lipids. ${ }^{4}$ These folate dependent functions are required for DNA synthesis and repair, control of gene expression and many other biological processes of fundamental importance for cell division and embryo development. ${ }^{5,6}$

Folate deficiency (genetically determined or due to dietary restriction) leads to higher frequency of uracil misincorporation into DNA, disruption of nucleic acid integrity, slower DNA replication and an increased risk of chromosome breakage. Insufficient folate or folic acid intake has also been shown to negatively affect specific reproductive functions; it has a detrimental effect on many processes involved in oocyte development, acquisition of endometrial receptivity, embryo implantation and also in the maintenance of pregnancy. ${ }^{7-10}$

Many variations in genes involved in folate metabolism have been identified.

In terms of prevalence and impact, genetic variations affecting the MTHFR are among the most biologically important. MTHFR is a key enzyme that plays an important role in catalyzing the conversion of 5, 10methylenetetrahydrofolate into 5methylenetetrahydrofolate, the predominant circulating form of folate. This molecule provides the single carbon needed for the synthesis of nucleotides, the re-methylation 
of homocysteine to methionine, the synthesis of $\mathrm{S}$ adenosylmethionine and the methylation of DNA, proteins, neurotransmitters and phospholipids. ${ }^{11,12}$

The most investigated are SNPs at the mRNA positions 677 (rs1801133) and 1298 (rs1801131). The wellcharacterised MTHFR c.677C $>\mathrm{T}$ transition, which results in an alanine to valine substitution (p.Ala222Val) in the predicted catalytic domain of MTHFR, renders the enzyme thermolabile and leads to a reduction in MTHFR activity. Homozygous and heterozygous individuals have in vitro MTHFR activity reduced by about $70 \%$ and $35 \%$, respectively. ${ }^{13}$ Homozygosity for the $677 \mathrm{~T}$ allele is associated with elevated circulating homocysteine in some individuals, predominantly those who have a low plasma folate level. ${ }^{14}$ In these individuals, the level of plasma homocysteine can be lowered by folic acid supplementation. ${ }^{15}$ The other common polymorphism in the MTHFR gene, c.1298A $>\mathrm{C}$ transversion, results in a glutamate to alanine substitution (p.Glu429Ala) within a presumed regulatory domain of MTHFR. ${ }^{16,17}$ The $1298 \mathrm{C}$ allele leads to decreased enzyme activity, although to a lesser extent than the $677 \mathrm{~T}$ allele. Individuals who are homozygous for the $1298 \mathrm{C}$ allele have about a $40 \%$ reduction in enzyme activity in vitro, but do not appear to have higher plasma homocysteine levels than controls. ${ }^{16-18}$ In few studies results demonstrated that maternal MTHFR c.1298A >C genotype strongly influences the likelihood of a pregnancy occurring, with the $1298 \mathrm{C}$ allele being significantly overrepresented amongst women who have undergone several unsuccessful assisted reproductive treatments also parental MTHFR genotypes were shown to affect the production of aneuploid embryos, indicating that MTHFR is one of the few known human genes with the capacity to modulate rates of chromosome abnormality. ${ }^{26}$
However, individuals who are compound heterozygous for the $677 \mathrm{~T}$ and the $1298 \mathrm{C}$ alleles (MTHFR c.677C/T plus c.1298A/C genotype) have a 40-50\% reduction in enzyme activity in vitro and a biochemical profile similar to that seen among 677T homozygotes, with increased homocysteine and decreased folate levels. The c.1298A>C polymorphism by itself may have clinically important effects under conditions of low folate intake or during times of high folate requirements such as pregnancy and embryogenesis. ${ }^{16}$ Some authors have reported an association of certain genotypes with an increased risk of miscarriage, a potential consequence of poor vascularization of the placental area of individuals carrying minor alleles. ${ }^{19-22}$ Others have described a link between c.677C $>\mathrm{T}$ and c. $1298 \mathrm{~A}>\mathrm{C}$ polymorphisms and the likelihood of aneuploid conceptions, pointing out the possible influence of MTHFR on chromosome nondisjunction and other processes involved in chromosome segregation. ${ }^{22-25}$ More recent reports have explored the impact of these polymorphisms in patients undergoing IVF treatment, suggesting an influence of some MTHFR variants on embryo implantation. ${ }^{27,28}$ There was still a need of analysis of the genotypes of individuals affected by fertility problems and of the embryos they produce was required in order to obtain a better understanding of the effects of MTHFR gene variants on reproduction in general and on assisted reproduction in particular.

\section{CASE SERIES}

Basic history and investigations are discussed in a tabular form.

Table 1: History of all three patients.

\begin{tabular}{|c|c|c|c|c|c|}
\hline $\begin{array}{l}\text { Patient's } \\
\text { name }\end{array}$ & Age & Marital status & Occupation & Menstrual history & Obstetric history \\
\hline AAP & 32 & $\begin{array}{l}\text { Married since } 9 \\
\text { years } \\
\text { (AML } 4 \text { years), } \\
\text { non- } \\
\text { consanguineous } \\
\text { marriage }\end{array}$ & $\begin{array}{l}\text { Interior } \\
\text { Designer }\end{array}$ & $\begin{array}{l}\text { Menarche } 13 \text { years } \\
\text { PrMC- } \\
\text { 3-4 days/1-2 pads/day/ } \\
\text { irregular since } 6 \text { months } \\
\text { PaMC 3-4 days/30 } \\
\text { days/RMPL }\end{array}$ & $\begin{array}{l}\text { A2 } \\
\text { A1-6WGA/natural conception/ } \\
\text { early pregnancy failure/dilatation } \\
\text { and evacuation done } \\
\text { A2-6WGA/natural } \\
\text { conception/missed } \\
\text { abortion/evacuated by medical } \\
\text { method. }\end{array}$ \\
\hline PM & 36 & $\begin{array}{l}\text { MS } 7 \text { years } \\
\text { AML } 2 \text { years } \\
\text { non- } \\
\text { consanguineous } \\
\text { marriage }\end{array}$ & Housewife & $\begin{array}{l}\text { Menarche } 12 \text { years } \\
\text { PrMC- } \\
5 \text { days/1-2pads/day/25 } \\
\text { days, PaMC- } \\
5 \text { days/1-2 pads/day/25 } \\
\text { days }\end{array}$ & $\begin{array}{l}\text { A2MTP2 } \\
\text { A1-6WGA/natural } \\
\text { conception/medical termination } \\
\text { i/v/o personal reasons/dilatation and } \\
\text { evacuation done } \\
\text { A2-6WGA/pregnancy diagnosed } \\
\text { with urine pregnancy test/natural } \\
\text { conception/early pregnancy } \\
\text { failure/evacuated by medical } \\
\text { method. } \\
\text { A3-12 WGA/natural conception/ } \\
\text { medical termination i/v/o raised }\end{array}$ \\
\hline
\end{tabular}




\begin{tabular}{|c|c|c|c|c|c|}
\hline $\begin{array}{l}\text { Patient's } \\
\text { name }\end{array}$ & Age & Marital status & Occupation & Menstrual history & Obstetric history \\
\hline & & & & & $\begin{array}{l}\text { nt/dilatation and evacuation done/ } \\
\text { product of conception tested } \\
\text { positive for trisomy } 13 \\
\text { A4- } 8 \text { WGA/natural } \\
\text { conception/missed } \\
\text { abortion/dilatation and evacuation } \\
\text { done. }\end{array}$ \\
\hline SPJ & 27 & $\begin{array}{l}\text { MS } 3 \text { years } \\
\text { AML } 3 \text { years } \\
\text { non - } \\
\text { consanguineous } \\
\text { marriage }\end{array}$ & Housewife & $\begin{array}{l}\text { Menarche - } 12 \text { years } \\
\text { PrMC- } \\
\text { 3-4 days/ 1-2pads/ day/ } \\
\text { 30 days } \\
\text { PaMC- } \\
\text { 3-4 days/ 1-2pads/ day/ } \\
\text { 30 days }\end{array}$ & $\begin{array}{l}\text { A2 } \\
\text { A1-6WGA/ natural conception/twin } \\
\text { pregnancy/one twin vanishing and } \\
\text { other missed abortion/dilatation and } \\
\text { evacuation done/POC karyotyping - } \\
\text { normal } \\
\text { A2-chemical pregnancy/natural } \\
\text { conception/early pregnancy } \\
\text { failure/spontaneously aborted. }\end{array}$ \\
\hline
\end{tabular}

MS-married since, AML-active married life , PrMC-present menstrual cycle, PaMC-past menstrual cycle, A-abortion , WGA-weeks gestational age, MTP-medical termination of pregnancy, POC-products of conception.

Table 2: History of all three patients (continued).

\begin{tabular}{|c|c|c|c|c|c|c|}
\hline $\begin{array}{l}\text { Patient's } \\
\text { name }\end{array}$ & $\begin{array}{l}\text { Contraception } \\
\text { history }\end{array}$ & $\begin{array}{l}\text { Sexual } \\
\text { history }\end{array}$ & Social history & $\begin{array}{l}\text { Past medical } \\
\text { and surgical } \\
\text { history }\end{array}$ & $\begin{array}{l}\text { Family } \\
\text { history }\end{array}$ & $\begin{array}{l}\text { Investigations } \\
\text { and treatment } \\
\text { done at other } \\
\text { ART center }\end{array}$ \\
\hline AAP & $\begin{array}{l}\text { History of use } \\
\text { of barrier } \\
\text { contraception } \\
\text { for } 4 \text { years } \\
\text { after marriage }\end{array}$ & $\begin{array}{l}\text { Coital } \\
\text { frequency } \\
\text { twice a } \\
\text { week; no } \\
\text { history of } \\
\text { coital } \\
\text { difficulties }\end{array}$ & $\begin{array}{l}\text { Nonalcoholic, } \\
\text { nonsmoker, } \\
\text { stress factor+ }\end{array}$ & $\begin{array}{l}\text { Known case of } \\
\text { hypothyroidism } \\
\text { on } 25 \mathrm{ug} \\
\text { levothyroxine }\end{array}$ & $\begin{array}{l}\text { Father- } \\
\text { hypertensive, } \\
\text { mother- } \\
\text { diabetic }\end{array}$ & $\begin{array}{l}2 \text { cycles of } \\
\text { ovulation } \\
\text { induction with T. } \\
\text { letrozole, no } \\
\text { dominant follicle } \\
\text { was formed. } \\
\text { Patient was } \\
\text { advised } \\
\text { thrombophilia } \\
\text { profile i/v/o } \\
\text { recurrent } \\
\text { pregnancy losses. } \\
\text { MTHFR 1298 A> } \\
\text { C heterozygote } \\
\text { variant present in } \\
\text { patient. } \\
\text { protein C, protein } \\
\text { S, S. } \\
\text { homocysteine, } \\
\text { antithrombin 3, } \\
\text { ACA, LA: } \\
\text { Negative } \\
\text { ANA-Weakly } \\
\text { positive }\end{array}$ \\
\hline PM & $\begin{array}{l}\text { History of use } \\
\text { of barrier } \\
\text { contraception } \\
\text { for } 5 \text { years } \\
\text { after marriage }\end{array}$ & $\begin{array}{l}\text { Coital } \\
\text { frequency } \\
\text { twice a } \\
\text { week; no } \\
\text { history of } \\
\text { coital } \\
\text { difficulties }\end{array}$ & $\begin{array}{l}\text { Nonalcoholic, } \\
\text { nonsmoker, } \\
\text { stress factor+ }\end{array}$ & Not significant & $\begin{array}{l}\text { Not } \\
\text { significant }\end{array}$ & None \\
\hline
\end{tabular}




\begin{tabular}{|c|c|c|c|c|c|c|}
\hline $\begin{array}{l}\text { Patient's } \\
\text { name }\end{array}$ & $\begin{array}{l}\text { Contraception } \\
\text { history }\end{array}$ & $\begin{array}{l}\text { Sexual } \\
\text { history }\end{array}$ & Social history & $\begin{array}{l}\text { Past medical } \\
\text { and surgical } \\
\text { history }\end{array}$ & $\begin{array}{l}\text { Family } \\
\text { history }\end{array}$ & $\begin{array}{l}\text { Investigations } \\
\text { and treatment } \\
\text { done at other } \\
\text { ART center }\end{array}$ \\
\hline SPJ & None & $\begin{array}{l}\text { Coital } \\
\text { frequency } \\
\text { twice a } \\
\text { week; no } \\
\text { history of } \\
\text { coital } \\
\text { difficulties }\end{array}$ & $\begin{array}{l}\text { Nonalcoholic, } \\
\text { nonsmoker, } \\
\text { stress factor+ }\end{array}$ & Not significant & $\begin{array}{l}\text { Not } \\
\text { significant }\end{array}$ & None \\
\hline
\end{tabular}

Table 3: Husband's history.

\begin{tabular}{|c|c|c|c|c|}
\hline $\begin{array}{l}\text { Patient's } \\
\text { name }\end{array}$ & Age & Occupation & Addiction & Semen analysis (at our centre) \\
\hline $\mathbf{A A P}$ & 32 & Interior designer & None & $\begin{array}{l}\text { Volume- } 2 \text { ml, sperm count- } 72 \\
\text { million/ml, motility-50\% (linear } \\
\text { progressive-45\%), morphology- } \\
48 \%, \text { DNA fragmentation index- } \\
\text { excellent }(10 \%)\end{array}$ \\
\hline $\mathbf{P M}$ & 37 & Businessman & None & $\begin{array}{l}\text { Volume- } 2.5 \mathrm{ml} \text {, sperm count- } 112 \\
\text { million/ml, total motility- } 80 \% \\
\text { (progressive motility } 60 \% \text { ), } \\
\text { morphology } 65 \%\end{array}$ \\
\hline SPJ & 28 & Businessman & None & $\begin{array}{l}\text { Volume-2 ml, sperm count-56 } \\
\text { million/ml, total motility-60\% } \\
\text { (progressive motility } 40 \% \text { ), } \\
\text { morphology-5\%, DNA } \\
\text { fragmentation index-15\% } \\
\text { excellent }\end{array}$ \\
\hline
\end{tabular}

Table 4: Investigations done at our center.

\begin{tabular}{|c|c|c|c|c|c|c|c|c|c|}
\hline $\begin{array}{l}\text { Patient's } \\
\text { name }\end{array}$ & $\mathbf{H b}$ & FSH & $\mathbf{L H}$ & $\mathbf{A M H}$ & PRL & TSH & $\begin{array}{l}\text { Thrombophilia } \\
\text { profile }\end{array}$ & Karyotype & USG-TVS \\
\hline AAP & 12 & 5.5 & 7 & 4.8 & 17 & 4.8 & $\begin{array}{l}\text { MTHFR } 1298 \\
\text { A>C; } \\
\text { heterozygote } \\
\text { variant present } \\
\text { in patient; ANA } \\
\text { weakly positive } \\
\text { (patient had } \\
\text { done at other } \\
\text { centre) }\end{array}$ & $\begin{array}{l}\text { Husband-46 } \\
\text { XY+13 } \\
\text { ps+(normal } \\
\text { polymorphic } \\
\text { variant) } \\
\text { Wife- } \\
46 \mathrm{XX}, 9 \mathrm{qh}+ \\
\text { (normal } \\
\text { polymorphic } \\
\text { variant) }\end{array}$ & $\begin{array}{l}\text { Uterus Av } 5.6 \times 3.7 \\
\mathrm{~cm} \\
\text { ET- } 8.6 \mathrm{~mm} \\
\text { RO- } 4 \times 2.25 \times 3.7 \mathrm{~cm}, \\
\text { AFC } 26 \\
\text { LO- } 3.5 \times 3 \times 3 \mathrm{~cm}, \\
\text { AFC } 30 \\
\text { Bilateral PCOS }\end{array}$ \\
\hline PM & 11 & 7 & 5 & 1.37 & 9.3 & 1.37 & $\begin{array}{l}\text { MTHFR } 677 \\
\text { C>T, } 1298 \text { A> } \\
\text { C heterozygote } \\
\text { variant present } \\
\text { in patient. } \\
\text { protein C, } \\
\text { protein S, S. } \\
\text { homocysteine, } \\
\text { antithrombin 3, } \\
\text { LA-negative }\end{array}$ & $\begin{array}{l}\text { Husband-46 XY } \\
\text { Wife-46 XX }\end{array}$ & $\begin{array}{l}\text { Day } 2 \text { USG uterus } \\
\text { AV } 7 \times 3.8 \mathrm{~cm} \\
\text { ET- } 3 \mathrm{~mm} \\
\text { RO- } 4 \times 2.25 \times 3.1 \mathrm{~cm} \\
\text { AFC } 8 \\
\text { LO- } 3.5 \times 3 \times 3 \mathrm{~cm} \mathrm{AFC} \\
7\end{array}$ \\
\hline
\end{tabular}




\begin{tabular}{|c|c|c|c|c|c|c|c|c|c|}
\hline $\begin{array}{l}\text { Patient's } \\
\text { name }\end{array}$ & $\mathbf{H b}$ & FSH & $\mathbf{L H}$ & $\mathbf{A M H}$ & PRL & TSH & $\begin{array}{l}\text { Thrombophilia } \\
\text { profile }\end{array}$ & Karyotype & USG-TVS \\
\hline & & & & & & & $\begin{array}{l}\text { ACA-weakly } \\
\text { positive } \\
\text { ANA-weakly } \\
\text { positive } \\
\text { (advised by us } \\
\text { due to RPL) }\end{array}$ & & \\
\hline SPJ & 11.8 & 4 & 3 & 4.2 & 19.7 & 2.6 & $\begin{array}{l}\text { Protein C, } \\
\text { protein S, APS } \\
\text { profile: negative } \\
\text { ANA-weakly } \\
\text { positive } \\
\text { (advised by us } \\
\text { due to RPL) }\end{array}$ & $\begin{array}{l}\text { Husband-46 X } \\
\text { inversion Y } \\
\text { (normal variant) } \\
\text { Wife-46 XX }\end{array}$ & $\begin{array}{l}\text { Uterus AV } 6.4 \times 3.4 \\
\mathrm{~cm} \\
\text { ET- } 7.5 \mathrm{~mm} \\
\text { RO- } 4 \times 2.25 \times 3.1 \mathrm{~cm} \\
\text { AFC } 25 \\
\text { LO- } 3 \times 4 \times 3 \mathrm{~cm} \mathrm{AFC} \\
30 \\
\text { B/L ovaries PCOS. }\end{array}$ \\
\hline
\end{tabular}

FSH-follicular stimulating hormone, LH-luteinizing hormone, TSH-thyroid stimulating hormone, AMH-anti-Mullerian hormone, PRL-prolactin, LA-lupus anticoagulant, ACA-anticardiolipin antibody, ET-endometrial thickness, AFC-antral follicle count, RO-right ovary, LO-left ovary, PCOS-polycystic ovarian syndrome.

\section{Case 1}

Patient AAP was advised either for HSG/diagnostic hysterolaproscopy followed by controlled ovarian stimulation+IUI (2-3 cycles) or IVF, patient directly opted for IVF.

As patient had history of two miscarriages IVF-ICSI+PGT was planned.

Patient was downregulated with tablet norethisterone $5 \mathrm{mg}$ thrice daily for 7 days and called on day 2 of menstrual cycle.

Stimulation was given with antagonist protocol and recombinant FSH, GnRH agonist was used as trigger.

Investigations on day of trigger were E2-3011, LH-0.74

Total 29 oocytes were retrieved (28 M2).

HSA on day of ICSI-count 102 million/ml, motility $50 \%$ (linear progressive-45\%), morphology $41 \%$.

Total fertilized was 24, total cleaved was 24 .

16 embryos were kept for blastocyst out of which 10 progressed-on 5 blastocyst PGS was done.

8 day 3 embryos and 5 blastocysts were vitrified.

PGT was performed and all blastocysts were normal and recommended for transfer.

Patient was monitored for OHSS and advised high oral intake.
HRT cycle was started (estrogen valerate $2 \mathrm{mg}$ TDS f/b injection micronized progesterone $\mathrm{P}+5$ ) and FET was done (ET $10.1 \mathrm{~mm}$ on day of transfer) with one PGT blastocystpatient tested negative.

2nd FET was done after 2 months with HRT cycle (same as above).

ET on day of transfer $9.7 \mathrm{~mm}, 2$ PGT blastocyst were transferred.

Patient tested positive-beta hCG was 254 .

\section{Case 2}

Patient PM was advised IVF i/v/o AMA with MTHFR positive status with bad obstetric history.

Patient was downregulated with tablet norethisterone $5 \mathrm{mg}$ thrice daily for 7 days and called on day 2 of menstrual cycle.

Stimulation was given with antagonist protocol and recombinant $\mathrm{FSH}$, rec hCG was used as trigger.

Investigations on day of trigger was 1004-3011, LH-0.766.

Total 8 oocytes were retrieved (4 M2).

HSA on day of ICSI-count 118 million/ml, motility 55\% (linear progressive-45\%), morphology $17 \%$.

Total fertilized were 4 , total were cleaved 4 .

4 day 3 embryos were vitrified.

Patient was planned for embryo pooling. 
2nd stimulation cycle was started after downregulation with tablet norethisterone for 7 days.

Patient was stimulated with ANTAGONIST PROTOCOL and recombinant $\mathrm{FSH}+\mathrm{hMG}(275+150$ stepped up to $300+150$ ), dual trigger was given with rec hCG and GnrH agonist (injection buserelin $0.5 \mathrm{mg}$ ).

Total oocytes retrieved were $12,7 \mathrm{M} 2$.

HSA on day of ICSI-count 120 million/ml, motility $52 \%$ (linear progressive-45\%), morphology $22 \%$.

Total fertilized was 6 , total cleaved was 6 .

All embryos were kept for blastocyst out of which 1 progressed to blastocyst on day 5 .

HRT cycle was started (estrogen valerate $2 \mathrm{mg}$ TDS f/b injection micronized progesterone $100 \mathrm{mg} \mathrm{OD}(\mathrm{P}+6)$ and FET was done (ET $10.1 \mathrm{~mm}$ on day of transfer) with twoday 6 blastocyst-patient tested positive, beta hCG 700 .

\section{Case 3}

Treatment history-i/v/o RPL hysteroscopy was planned.

Findings were short septum was present in uterine cavity, resection with scissors done.

Endometrial biopsy taken and sent for TB-PCR was negative.

Patient SPJ was advised ovulation induction+timed intercourse for 1-2 cycles.

1.1 cycle of OI given with tablet letrozole-patient was tested beta hCG positive (77).

Developed bleeding PV and spontaneously aborted.

Endometrial study and uterine artery Doppler were performed which showed normal findings.

I/v/o recurrent pregnancy losses-unexplained, patient opted for IVF.

Patient was downregulated with tablet norethisterone $5 \mathrm{mg}$ thrice daily for 7 days and called on day 2 of menstrual cycle.

Stimulation was given with antagonist protocol and recombinant $\mathrm{FSH}$, rec hCG was used as trigger.

Investigations on day of trigger-E2-1800, LH-0.66.

Total 9 oocytes were retrieved (9 M2).
HSA on day of ICSI-count 118 million/ml, motility 55\% (linear progressive-45\%), morphology $17 \%$.

Total fertilized was 8 , total cleaved was 8 .

Sequential fresh embryo transfer was done on D3- O2, D501

Beta hCG was 1309 and ectopic pregnancy in the right tube was found.

Expectant management was observed-beta hCG was followed up till it was less than 1 .

HRT cycle was started with tablet estradiol $2 \mathrm{mg}$ TDS f/b injection micronized progesterone $100 \mathrm{mg}$ once daily for 5 days.

Frozen embryo transfer-1 grade 1 blastocyst was done.

Patient tested positive-first beta hCG 41 with rising titres, TVS USG obstetrics was suggestive of intrauterine pregnancy of 5 weeks.

Patient developed bleeding PV-beta hCG was done with showed falling titres.

Repeat TVS-USG suggestive of missed abortion with empty uterine cavity.

Patient again conceived naturally after 3 months, beta CG 3540/TVS USG obstetrics s/o single live intrauterine pregnancy of 6.6 weeks.

Patient developed bleeding PV and follow up USG showed missed abortion.

Dilation and evacuation were done.

I/v/o 5 pregnancy losses ( 2 missed abortions and 3 early pregnancy failures) patient was advised MTHFR gene mutation testing-she tested homozygous positive for A1298C mutation.

A decision for surrogacy was taken-a healthy baby of $3 \mathrm{~kg}$ delivered at term.

\section{DISCUSSION}

The occurrence of MTHFR gene mutation in Indian population had not been widely studied.

All the more its role in recurrent pregnancy losses and assisted reproductive technique outcomes was yet to be concretely established in Indian population. The data on the exact role of MTHFR mutation on Indian female patients with recurrent miscarriage/RPL had been mostly lacking. ${ }^{1}$ 
MTHFR gene polymorphisms have been shown to be associated with vascular diseases, colon cancer, in pregnancy complications such as recurrent miscarriages, malformations in fetal development and non-disjunction of chromosomes. $^{2}$

In our case series of three patients, all three were positive for some different variant of MTHFR gene mutation. All three patients had history of recurrent pregnancy losses with other causes ruled out.

MTHFR gene mutation testing should be included after 3 missed abortions/pregnancy failures.

One patient opted for PGT and it was found that all the tested embryos in her case were euploid.

Transfer of an euploid embryo also resulted in miscarriage depicting that may be MTHFR gene mutation does play an important role in endometrial receptivity, implantation and placentogenesis. Also in case 3, there were multiple pregnancy failures in the patient and finally it was decided to go ahead with surrogacy, a full-term baby of $3 \mathrm{~kg}$ was born.

\section{CONCLUSION}

Our case series gives a new pathway to management of cases with recurrent pregnancy losses or recurrent IVF failures where testing for MTHFR gene mutation can open new gateways for further management strategies for a successful pregnancy outcome like PGT to rule out aneuploidy caused due to disturbance in folate metabolism as a consequence of MTHFR gene mutation and transfer of euploid embryos. Surrogacy in cases of recurrent pregnancy failures even with assisted reproductive technique. MTHFR gene mutation (both variants) and their relation to reproductive outcomes need more studies and large prospective randomized controlled trials in Indian population.

\section{Funding: No funding sources Conflict of interest: None declared Ethical approval: Not required}

\section{REFERENCES}

1. Gupta N, Gupta G, Chakrabarti S, Thangaraj K, Singh L, Chakravarty B. Mutation analysis of mthfr gene in indian women with unexplained recurrent miscarriages; folic acid supplementation improves pregnancy outcomes. Am J Stem Cell. 2019;14.

2. Schneider JA, Rees DC, Liu YT, Clegg JB. Worldwide distribution of a common methylenetetrahydrofolate reductase mutation. Am J Hum Genet.1998;62(5):1258-60.

3. Tamura T, Picciano MF. Folate and human reproduction. Am J Clin Nutr. 2006;83(5):993-1016.

4. Wagner CL. Biochemical role of folate in cellular metabolism. Folate in health and disease. In: Bailey
LB, eds. New York, NY: Marcel Dekker; 1995: 2342.

5. Obican SG, Finnell RH, Mills JL, Shaw GM, Scialli AR. Folic acid in early pregnancy: a public health success story. Faseb J 2010;24(11):4167-74.

6. Kim KC, Friso S, Choi SW. (2009). DNA methylation, an epigenetic mechanism connecting folate to healthy embryonic development and aging. J Nutr Biochem. 2009;20(12):917-26.

7. Gmyrek GB, Sozanski R, Jerzak M, Chrobak A, Wickiewicz D, Skupnik A, et al. Evaluation of monocyte 24 chemotactic protein-1 levels in peripheral blood of infertile women with endometriosis. Eur J Obstet Gynecol Reprod Biol. 2005;122(2):199-205.

8. Thaler CD, Epel D. Nitric oxide in oocyte maturation, ovulation, fertilization, cleavage and implantation: a little dab'll do ya. Curr Pharm Des. 2003;9(5):399409.

9. Hussein MR. Apoptosis in the ovary: molecular mechanisms. Hum Reprod Update. 2005;11(2):16277.

10. Forges T, Monnier-Barbarino P, Alberto JM, GueantRodriguez RM, Daval JL, Gueant JL. Impact of folate and homocysteine metabolism on human reproductive health. Hum Reprod Update. 2007;13(3):225-38.

11. Frosst P, Blom HJ, Milos R, Goyette P, Sheppard CA, Matthews RG, et al. A candidate genetic risk factor for vascular disease: a common mutation in methylenetetrahydrofolate reductase. Nat Genet. 1995;10(1):111-3.

12. Yamada K, Strahler JR, Andrews PC, Matthews RG. Regulation of human methylenetetrahydrofolate reductase by phosphorylation. Proc Natl Acad Sci U S A. 2005;102(30):10454-9.

13. Frosst P, Blom HJ, Milos R, Goyette P, Sheppard CA, Matthews RG, et al. A candidate genetic risk factor for vascular disease: a common mutation in methylenetetrahydrofolate reductase. Nat Genet. 1995;10(1):111-3.

14. Jacques PF, Bostom AG, Williams RR, Ellison RC, Eckfeldt JH, Rosenberg IH, et al. Relation between folate status, a common mutation in methylenetetrahydrofolate reductase, and plasma homocysteine concentrations. Circulation. 1996;93(1):7-9.

15. Malinow MR, Nieto FJ, Kruger WD, Duell PB, Hess DL, Gluckman RA, et al. The effects of folic acid supplementation on plasma total homocysteine are modulated by multivitamin use and methylenetetrahydrofolate reductase genotypes. Arterioscler Thromb Vasc Biol. 1997;17(6):1157-62.

16. Put NMJ, Gabreels F, Stevens EM, Smeitink JAM, Trijbels FJM, Eskes et al. A second common mutation in the methylenetetrahydrofolate reductase gene: An additional risk factor for neural-tube defects? Am J Hum Genet. 1998;62(5):1044-51.

17. Weisberg I, Tran P, Christensen B, Sibani S, Rozen R. A second genetic polymorphism in methylenetetrahydrofolate reductase (MTHFR) 
associated with decreased enzyme activity. Mol Genet Metab. 1998;64(3):169-72.

18. Chango A, Boisson F, Barbe F, Quilliot D, Droesch S, Pfister M, et al. The effect of 677C-->T and 1298A-$>\mathrm{C}$ mutations on plasma homocysteine and 5,10methylenetetrahydrofolate reductase activity in healthy subjects. Br J Nutr. 2000;83(6):593-6.

19. Nelen WL, Bulten J, Steegers EA, Blom HJ, Hanselaar AG, Eskes TK. Maternal homocysteine and chorionic vascularization in recurrent early pregnancy loss. Hum Reprod. 2000;15(4):954-60.

20. Nair RR, Khanna A, Singh K. MTHFR C677T polymorphism and recurrent early pregnancy loss risk in north Indian population. Reprod Sci. 2012;19(2):210-5.

21. Cao Y, Xu J, Zhang Z, Huang X, Zhang A, Wang J, et al. (2013). Association study between methylenetetrahydrofolate reductase polymorphisms and unexplained recurrent pregnancy loss: a metaanalysis. Gene. 2013;514(2):105-11.

22. Kim SY, Park SY, Choi JW, Kim J, Lee SY, Lim JH, et al. Association between MTHFR 1298A>C polymorphism and spontaneous abortion with fetal chromosomal aneuploidy. Am J Reprod Immunol. 2011;66(4):252-8.

23. James SJ, Pogribna M, Pogribny IP, Melnyk S, Hine RJ, Gibson JB, et al. Abnormal folate metabolism and mutation in the methylenetetrahydrofolate reductase gene may be maternal risk factors for Down syndrome. Am J Clin Nutr. 1999;70(4):495-501.

24. Hobbs CA, Sherman SL, Yi P, Hopkins SE, Torfs CP, Hine RJ, et al. (2000). Polymorphisms in genes involved in folate metabolism as maternal risk factors for Down syndrome. Am J Hum Genet. 2000;67(3):623-30.

25. Hassold TJ, Burrage LC, Chan ER, Judis LM, Schwartz S, James SJ, et al. Maternal folate polymorphisms and the etiology of human nondisjunction. Am J Hum Genet. 2001;69(2):434-9.

26. Enciso M, Sarasa J, Xanthopoulou L. Polymorphisms in the MTHFR gene influence embryo viability and the incidence of aneuploidy. Hum Genet. 2016;135:555-68.

27. Laanpere M, Altmae S, Kaart T, Stavreus-Evers A, Nilsson TK, Salumets A. Folate-metabolizing gene variants and pregnancy outcome of IVF. Reprod Biomed Online. 2011;22(6):603-14.

28. Soldo V, Cutura N, Zamurovic M. Defect of methylenetetrahydrofolate reductase in a patient with ten habitual miscarriages: a case report. Clin Exp Obstet Gynecol. 2012;39(4):556-8.

Cite this article as: Singh PN, Raghav M, Singh P, Viradiya V. 5, 10-methylenetetrahydrofolate reductase gene mutation and reproductive outcome: how much do we know? A case series in Indian population. Int J Reprod Contracept Obstet Gynecol 2022;11:908-15. 